\title{
The extended stellar substructures of four metal-poor globular clusters in the Galactic bulge
}

\author{
Sang-Hyun Chun ${ }^{1}$ and Young-Jong Sohn ${ }^{2}$ \\ ${ }^{1}$ Yonsei University Observatory, 120-749 Seoul, Korea \\ email: shchun@galaxy.yonsei.ac.kr \\ ${ }^{2}$ Department of Astronomy, Yonsei University, 120-749, Seoul, Korea \\ email: sohnyj@yonsei.ac.kr
}

\begin{abstract}
We investigated the stellar density substructures around four metal-poor globular clusters (NGC 6266, NGC 6626, NGC 6642, and NGC 6723) in the Galactic bulge. Wide-field near-infrared $\left(J H K_{s}\right)$ imaging data were obtained from WFCAM of UKIRT telescope. Field stars contamination around the globular clusters was reduced by using a statistical weighted filtering algorithm. Tidal stripping stellar substructures in the form of tidal tail (NGC 6266 and NGC 6626) or small density lobes/chunk (NGC 6642 and NGC 6723) were found around the four globular clusters in the two-dimensional density contour maps. We also find the overdensity features, which deviate from the theoretical models, in the outer region of radial density profiles. The observed results imply that the four globular clusters have experienced a strong tidal force or the bulge/disk shock effect of the Galaxy.
\end{abstract}

Keywords. Globular clusters, Tidal tail

\section{Introduction}

According to the hierarchical scenario of the Galaxy formation, galaxies are developed by merging and accretion of small fragments like dwarf galaxies and globular clusters (Moore et al. 1999, Bullock et al. 2001). In this process, accreted satellite systems are disrupted by tidal force and shock, and then leave stellar substructures such as tidal tails and tidal streams (Bullock \& Johnston 2005). Thus, the study of the stellar substructures can provide the observational evidence for merging and accretion of satellites. We investigated the tidal substructures around four metal-poor globular clusters in the bulge region.

\section{Extended stellar substructures}

We obtained wide-field $\left(45^{\prime} \times 45^{\prime}\right)$ near-infrared photometric data for four globular clusters (NGC 6266, NGC 6626, NGC 6642, and NGC 6723) using the WFCAM array of UKIRT. A statistical weighted filtering algorithm (Chun et al. 2012, Grillmair et al. 1995, Odenkirchen et al. 2003) was applied to the CMDs of the clusters in order to minimize the field star contamination.

Figure 1 shows the two-dimensional contour maps and radial density profile of the four globular clusters. We find that NGC 6266 and NGC 6626 show extended stellar substructures beyond tidal radius, while NGC 6642 and NGC 6723 have small density chunks or lobes near tidal radius. The extended stellar substructures seem to be aligned with the proper motion direction of cluster and the direction of the Galactic center. 

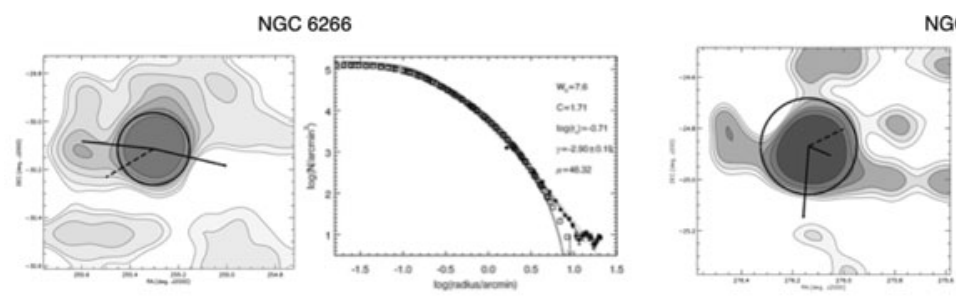

NGC 6626

NGC 6642
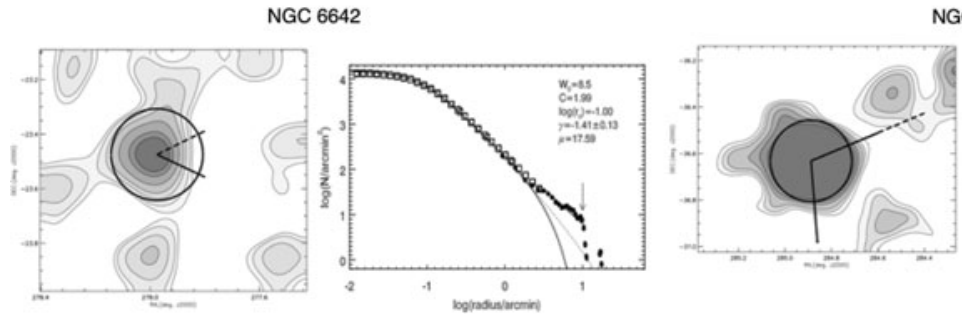

NGC 6723
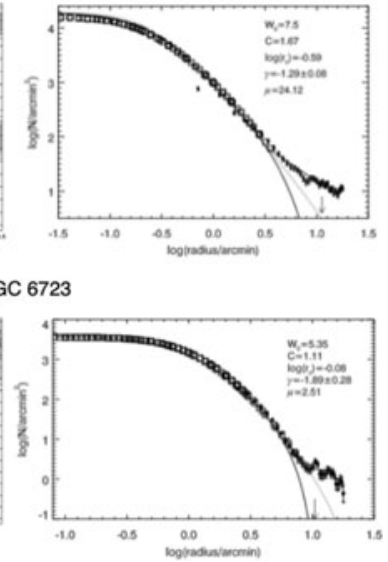

Figure 1. Iso-density maps and radial profiles for the four globular clusters. In contour maps: Arrows indicate the proper motion of cluster. The solid line and dashed lines indicate the direction of the Galactic center and that perpendicular to the Galactic plane, respectively. In radial profiles: the solid curve and dotted curved show the King model and Wilson models. Open rectangules represent the surface brightness profile of Trager et al. (1995). Filled circles are our measurements, and arrows indicate the tidal radius of the clusters.

The distorted stellar substructures are also well represented in the radial profile as an overdensity feature which departs from theoretical King (King 1966) and Wilson (Wilson 1975) models at the outer region of the clusters.

\section{Discussion}

We found that tidally extended stellar features are present in the vicinity of four metal-poor globular clusters. The stellar substructures seem to be associated with the interaction of the Galactic tidal force and proper motion of clusters. Our results indicate that strong tidal force or bulge/disk shock have affected the shape of the four globular clusters in the bulge region, and provide further constraints on the formation of the globular clusters in the bulge region.

\section{References}

Bullock, J. S., Kratsov, A. V., \& Weinberg, D. H. 2001, ApJ, 548, 33

Bullock, J. S. \& Johnston, K. V. 2005, ApJ, 635, 931

Chun, S.-H., Kim, J.-W., Kim, M.-J., et al. 2012, AJ, 144, 26

Grillmair, C. J., Freeman, K. C., Irwin, M., \& Quinn, P. J. 1995, AJ, 109, 2553

King, I. R. 1966, $A J, 71,64$

Moore, B., Ghigna, S., Governato, F., et al. 1999, ApJ, 524, L19

Odenkirchen, M., Grebel, E. K., Dehnen, W., et al. 2003, AJ, 126, 2385

Trager, S. C., King, Ivan R., \& Djorgovski, S. 1995, AJ, 109, 218

Wilson, C. P. 1975, AJ, 80, 175 